\title{
Characterization of Some Aggregation Functions Stable for Positive Linear Transformations
}

\author{
Jean-Luc Marichal* $^{*} \quad$ Pierre Mathonet $^{\dagger} \quad$ Eric Tousset $^{\dagger}$ \\ Revised version, February 28, 1997
}

\begin{abstract}
This paper deals with the characterization of some classes of aggregation functions often used in multicriteria decision making problems. The common properties involved in these characterizations are "increasing monotonicity" and "stability for positive linear transformations". Additional algebraic properties related to associativity allow to completely specify the functions.
\end{abstract}

Keywords: aggregation functions; interval scale; invariance; algebraic properties of aggregation functions; fuzzy multiple criteria decision making.

\section{Introduction}

Synthesizing judgments is an important part of multiple criteria decision making methods. The most typical situation concerns individuals who form quantifiable judgments about a measure of an object (weight, length, area, height, volume, importance or other attributes, for instance in the framework of a hierarchy)(see [3, 4]) or quantifiable judgments on pairs of alternatives along each criterion. In the latter case, the judgments are very often expressed with the help of fuzzy preference relations (see $[8,9])$.

In order to reach a consensus (overall opinion) on these jugdments, classical aggregation functions have been proposed: arithmetic means, geometric means, root-power means and many others. Of course, given such an aggregation function, we can ask for a motivation of its use, i.e. for natural, reasonable assumptions which lead to this function. Conversely, we can specify some assumptions (called axioms or properties) and determine all the aggregation functions satisfying these. This is the topic with which we deal here.

This paper aims at describing the family of all aggregation functions fulfilling three specific properties. The first two are increasing monotonicity and stability for the same transformations of interval scales in the sense of the theory of measurement (see [15]), i.e. stability for positive linear transformations (we refer to the corresponding functional equation in $[5,6]$ where the arithmetic mean is characterized). The third property is chosen among well-known algebraic properties such as associativity, decomposability and bisymmetry. See Section 3 for details.

We make a distinction between aggregation functions having a fixed number of arguments and aggregation functions defined for all number of arguments (aggregators). Section 4 is devoted to characterizations of aggregation functions with a fixed number of arguments whereas Section 5 presents characterizations of aggregators.

\footnotetext{
*Ecole d'Administration des Affaires, Université de Liège, Boulevard du Rectorat 7 - B31, B-4000 Liège, Belgium. Email: jl.marichal[at]ulg.ac.be

${ }^{\dagger}$ Institut de Mathématique, Université de Liège, Avenue des Tilleuls 15 - D1, B-4000 Liège, Belgium. Email: mathonet[at]math.ulg.ac.be
} 


\section{Basic definitions}

We first want to define the concept of aggregation function. In this paper, $\mathbb{N}^{*}$ denotes the set of strictly positive integers and $\mathbb{R}$ the set of real numbers. Moreover, we assume that the information to be aggregated consists of numbers belonging to the interval $[0,1]$ as required in most applications. In Section 7, we show that this assumption can be weakened.

Definition 2.1 Let $m \in \mathbb{N}^{*}$. An aggregation function $M^{(m)}$ defined on $[0,1]^{m}$ is a real valued function of $m$ arguments:

$$
M^{(m)}:[0,1]^{m} \rightarrow \mathbb{R}:\left(x_{1}, \ldots, x_{m}\right) \rightarrow M^{(m)}\left(x_{1}, \ldots, x_{m}\right) .
$$

For instance, the arithmetic mean function defined by

$$
A M^{(m)}\left(x_{1}, \ldots, x_{m}\right)=\frac{1}{m} \sum_{i=1}^{m} x_{i} \forall\left(x_{1}, \ldots, x_{m}\right) \in[0,1]^{m}
$$

is an aggregation function defined on $[0,1]^{m}$.

Definition 2.2 An aggregator $M$ defined on $\bigcup_{m \geq 1}[0,1]^{m}$ is a sequence $\left(M^{(m)}\right)_{m \in \mathbb{N}^{*}}$ whose mth element is an aggregation function $M^{(m)}$ defined on $[0,1]^{m}$ :

$$
M: \bigcup_{m \geq 1}[0,1]^{m} \rightarrow \mathbb{R}:\left(x_{1}, \ldots, x_{m}\right) \rightarrow M^{(m)}\left(x_{1}, \ldots, x_{m}\right) .
$$

For instance, the arithmetic mean aggregator is $A M=\left(A M^{(m)}\right)_{m \in \mathbb{N}^{*}}$.

Let $m \in \mathbb{N}^{*}$. We consider a discrete set of $m$ elements $N_{m}=\{1, \ldots, m\}$, which could be players of a cooperative game, criteria, attributes or voters in a decision making problem.

In order to avoid heavy notations, we introduce the following terminology. It will be used all along this paper.

- $\mathrm{II}:=[0,1]$

- For all $k \in \mathbb{N}^{*}$ and all $x \in \mathbb{I}, k \cdot x:=\underbrace{x, \ldots, x}_{k}$

- For all $N \subseteq N_{m}$, the characteristic vector of $N$ in $\{0,1\}^{m}$ is defined by

$$
e_{N}^{(m)}:=\left(x_{1}, \ldots, x_{m}\right) \in\{0,1\}^{m} \text { with } x_{i}=1 \Leftrightarrow i \in N .
$$

Of course, the $e_{N}^{(m)}$ 's $\left(N \subseteq N_{m}\right)$ are the $2^{m}$ vertices of the hypercube $\mathbb{I}^{m}$. We also introduce the complementary characteristic vector of $N \subseteq N_{m}$ :

$$
\bar{e}_{N}^{(m)}:=e_{N_{m} \backslash N}^{(m)} .
$$

Then we set

$$
\theta_{N}^{(m)}:=M^{(m)}\left(e_{N}^{(m)}\right) \text { and } \bar{\theta}_{N}^{(m)}:=M^{(m)}\left(\bar{e}_{N}^{(m)}\right) .
$$

The expressions $e_{\{i\}}^{(m)}, \bar{e}_{\{i\}}^{(m)}, \theta_{\{i\}}^{(m)}, \bar{\theta}_{\{i\}}^{(m)}$ will be denoted $e_{i}^{(m)}, \bar{e}_{i}^{(m)}, \theta_{i}^{(m)}, \bar{\theta}_{i}^{(m)}$ respectively.

- Given a vector $\left(x_{1}, \ldots, x_{m}\right) \in \mathbb{I}^{m}$, let $x_{(1)}, \ldots, x_{(m)}$ denote the elements of this vector sorted in increasing order: that is, $x_{(1)} \leq \ldots \leq x_{(m)}$. 


\section{$3 \quad$ Aggregation properties}

As mentioned in the introduction, if we want to obtain a reasonable or satisfactory aggregation, any aggregation function should not be used. In order to evacuate the "undesirable" functions, we can adopt an axiomatic approach and impose that these functions fulfil some selected properties. Such properties can be divided into three categories: natural properties, stability properties and algebraic properties.

\subsection{Natural properties}

Definition 3.1 The aggregation function $M^{(m)}$ defined on $\mathbb{I}^{m}$ is

- symmetric (Sy) if $M^{(m)}$ is a symmetric function on $\mathbb{I}^{m}$, i.e. if, for all permutations $\sigma$ of $N_{m}$ and all $\left(x_{1}, \ldots, x_{m}\right) \in \mathbb{I}^{m}$, we have

$$
M^{(m)}\left(x_{1}, \ldots, x_{m}\right)=M^{(m)}\left(x_{\sigma(1)} \ldots, x_{\sigma(m)}\right) .
$$

- increasing (In) if $M^{(m)}$ is increasing in each argument, i.e. if, for all $i \in N_{m}$ and all $x_{1}, \ldots, x_{m}, x_{i}^{\prime} \in \mathbb{I I}$, we have

$$
x_{i}<x_{i}^{\prime} \Rightarrow M^{(m)}\left(x_{1}, \ldots, x_{i}, \ldots, x_{m}\right) \leq M^{(m)}\left(x_{1}, \ldots, x_{i}^{\prime}, \ldots, x_{m}\right) .
$$

- compensative (Comp) if, for all $\left(x_{1}, \ldots, x_{m}\right) \in \mathbb{I}^{m}$,

$$
\min \left(x_{1}, \ldots, x_{m}\right) \leq M^{(m)}\left(x_{1}, \ldots, x_{m}\right) \leq \max \left(x_{1}, \ldots, x_{m}\right) .
$$

- idempotent (I) if, for all $x \in \mathbb{I}$,

$$
M^{(m)}(m \cdot x)=x
$$

It should be noted that any compensative aggregation function defined on $\mathbb{I}^{m}$ necessarily takes its values in II. Moreover, we have the following result which can easily be checked:

Proposition 3.1 For every aggregation function $M^{(m)}$ defined on $\mathbb{I}^{m}$,

$$
\begin{array}{ll}
\text { (i) } & (\text { Comp }) \Rightarrow(I) \\
\text { (ii) } & (\text { In }, I) \Rightarrow(\text { Comp })
\end{array}
$$

The properties mentioned in Definition 3.1 can be adapted to aggregators as follows.

Definition 3.2 The aggregator $M$ defined on $\bigcup_{m \geq 1} \mathbb{I}^{m}$ fulfils (Sy) (resp. (In), (Comp), (I)) if, for all $m \in \mathbb{N}^{*}$, mth aggregation function $M^{(m)}$ fulfils (Sy) (resp. (In), (Comp), (I)).

\subsection{Stability properties}

Definition 3.3 The aggregation function $M^{(m)}$ defined on $\mathbb{I}^{m}$ is

- stable for the admissible similarities (SSI) if

$$
M^{(m)}\left(r x_{1}, \ldots, r x_{m}\right)=r M^{(m)}\left(x_{1}, \ldots, x_{m}\right)
$$

for all $\left(x_{1}, \ldots, x_{m}\right) \in \mathbb{I}^{m}$ and all $r>0$ such that $r x_{i} \in \mathbb{I}$ for all $i \in N_{m}$.

- stable for the admissible translations (STR) if

$$
M^{(m)}\left(x_{1}+t, \ldots, x_{m}+t\right)=M^{(m)}\left(x_{1}, \ldots, x_{m}\right)+t
$$

for all $\left(x_{1}, \ldots, x_{m}\right) \in \mathbb{I}^{m}$ and all $t \in \mathbb{R}$ such that $x_{i}+t \in \mathbb{I}$ for all $i \in N_{m}$. 
- stable for the admissible positive linear transformations (SPL) if

$$
M^{(m)}\left(r x_{1}+t, \ldots, r x_{m}+t\right)=r M^{(m)}\left(x_{1}, \ldots, x_{m}\right)+t
$$

for all $\left(x_{1}, \ldots, x_{m}\right) \in \mathbb{I}^{m}$ and all $r>0, t \in \mathbb{R}$ such that $r x_{i}+t \in \mathbb{I}$ for all $i \in N_{m}$.

- stable for the standard negation $\mathrm{N}(\mathrm{SSN})$ if

$$
M^{(m)}\left(1-x_{1}, \ldots, 1-x_{m}\right)=1-M^{(m)}\left(x_{1}, \ldots, x_{m}\right)
$$

for all $\left(x_{1}, \ldots, x_{m}\right) \in \mathbb{I}^{m}$.

The use of stability properties supposes that the values to be aggregated are given according to some scale type as defined by Roberts [15]. Note that some characterization theorems were obtained by Nagumo [14] for (SSI) and (STR) and by Silvert [16] for (SSN) (see also [9, pp.117126] and [13]). The next result gives some relations between stability properties. These relations will be useful in the sequel.

Proposition 3.2 For all aggregation function $M^{(m)}$ defined on $\mathbb{I}^{m}$, we have

$$
\begin{aligned}
\text { (i) } & (S S I, S T R) \Leftrightarrow(S P L) \\
\text { (ii) } & (S S I) \Rightarrow M^{(m)}(m \cdot 0)=0 \\
\text { (iii) } & (S P L) \Rightarrow(I) \\
\text { (iv) } & (S S I, S S N) \Rightarrow(S P L)
\end{aligned}
$$

Proof. (i) Trivial.

(ii) It suffices to consider $\left(x_{1}, \ldots, x_{m}\right)=(m \cdot 0)$.

(iii) It suffices to use $(i)$ and $(i i)$.

(iv) By $(i)$, it suffices to show that $M^{(m)}$ fulfils (STR). Let $\left(x_{1}, \ldots, x_{m}\right) \in \mathbb{I}^{m}$ and $t \in[-1,1]$ such that $x_{i}+t \in \mathbb{I} \forall i \in N_{m}$. Let us prove that

$$
M^{(m)}\left(x_{1}+t, \ldots, x_{m}+t\right)=M^{(m)}\left(x_{1}, \ldots, x_{m}\right)+t .
$$

By $(i i)$ and (SSN) we have $M^{(m)}(m \cdot 1)=1-M^{(m)}(m \cdot 0)=1$. So we can assume that $t$ lies in the open interval $(-1,1)$. For all $i \in N_{m}$ set $y_{i}=\frac{x_{i}}{1-t}$. We then have $y_{i} \in \mathbb{I} \forall i \in N_{m}$ and

$$
\begin{aligned}
& M^{(m)}\left(x_{1}, \ldots, x_{m}\right) \stackrel{(S S I)}{=}(1-t) M^{(m)}\left(y_{1}, \ldots, y_{m}\right) \\
& \stackrel{(S S N)}{=}(1-t)-(1-t) M^{(m)}\left(1-y_{1}, \ldots, 1-y_{m}\right) \\
& \stackrel{(S S I)}{=}(1-t)-M^{(m)}\left[(1-t)-x_{1}, \ldots,(1-t)-x_{m}\right] \\
& \stackrel{(S S N)}{=}-t+M^{(m)}\left(x_{1}+t, \ldots, x_{m}+t\right) \text {. }
\end{aligned}
$$

It clearly turns out, by the previous proposition, that the condition " $r>0$ " in the statement of (SSI) or (SPL) can be replaced by " $r \geq 0$ " without any effect.

The next proposition characterizes the aggregation functions $M^{(m)}$ defined on $\mathbb{I}^{m}$ and satisfying (SPL). A similar characterization was obtained by Aczél and Roberts [5, p.220 (Case 5b)] in the case of aggregation functions $M^{(m)}$ defined on $\mathbb{R}^{m}$.

Proposition 3.3 An aggregation function $M^{(m)}$ defined on $\mathbb{I}^{m}$ fulfils (SPL) if and only if there exists an aggregation function $F^{(m)}$ defined on $\mathbb{I}^{m}$ such that, for all $\left(x_{1}, \ldots, x_{m}\right) \in \mathbb{I}^{m}$, we have

$$
M^{(m)}\left(x_{1}, \ldots, x_{m}\right)=\left\{\begin{array}{l}
x \text { if }\left(x_{1}, \ldots, x_{m}\right)=(x, \ldots, x) \\
\left(x_{(m)}-x_{(1)}\right) F^{(m)}\left[\frac{x_{1}-x_{(1)}}{x_{(m)}-x_{(1)}}, \ldots, \frac{x_{m}-x_{(1)}}{x_{(m)}-x_{(1)}}\right]+x_{(1)} \text { otherwise. }
\end{array}\right.
$$


Proof. (Sufficiency). Trivial.

(Necessity). It suffices to consider $F^{(m)}=M^{(m)}$.

Definition 3.4 The aggregator $M$ defined on $\bigcup_{m \geq 1} \mathbb{I}^{m}$ fulfils (SSI) (resp. (STR), (SPL), (SSN)) if, for all $m \in \mathbb{N}^{*}$, the $m$ th aggregation function $M^{(m)}$ fulfils (SSI) (resp. (STR), (SPL), (SSN)).

This paper mostly concentrates on the characterization of aggregation functions and aggregators satisfying properties (In) and (SPL), as well as some additional properties such as (Sy), (SSN), or algebraic properties to be introduced next.

\subsection{Algebraic properties}

Definition 3.5 The aggregation function $M^{(m)}$ defined on $\mathbb{I}^{m}$ is

- associative (A) if $m=2$ and

$$
M^{(2)}\left(M^{(2)}\left(x_{1}, x_{2}\right), x_{3}\right)=M^{(2)}\left(x_{1}, M^{(2)}\left(x_{2}, x_{3}\right)\right)
$$

for all $\left(x_{1}, x_{2}, x_{3}\right) \in \mathbb{I}^{3}$.

- autodistributive (AD) if $m=2$ and

$$
\begin{aligned}
& M^{(2)}\left(x_{1}, M^{(2)}\left(x_{2}, x_{3}\right)\right)=M^{(2)}\left(M^{(2)}\left(x_{1}, x_{2}\right), M^{(2)}\left(x_{1}, x_{3}\right)\right), \\
& M^{(2)}\left(M^{(2)}\left(x_{1}, x_{2}\right), x_{3}\right)=M^{(2)}\left(M^{(2)}\left(x_{1}, x_{3}\right), M^{(2)}\left(x_{2}, x_{3}\right)\right)
\end{aligned}
$$

for all $\left(x_{1}, x_{2}, x_{3}\right) \in \mathbb{I}^{3}$.

- $\operatorname{bisymmetric~(B)~if~} m \geq 2$ and

$$
\begin{aligned}
& M^{(m)}\left(M^{(m)}\left(x_{11}, \ldots, x_{1 m}\right), \ldots, M^{(m)}\left(x_{m 1}, \ldots, x_{m m}\right)\right) \\
= & M^{(m)}\left(M^{(m)}\left(x_{11}, \ldots, x_{m 1}\right), \ldots, M^{(m)}\left(x_{1 m}, \ldots, x_{m m}\right)\right)
\end{aligned}
$$

for all square matrices

$$
X=\left(\begin{array}{ccc}
x_{11} & \cdots & x_{1 m} \\
\vdots & & \vdots \\
x_{m 1} & \cdots & x_{m m}
\end{array}\right) \in \mathbb{I}^{m \times m}
$$

Note that those definitions make sense only if $M^{(m)}$ takes its values in $\mathbb{I}$.

The properties mentioned in Definition 3.5 were investigated by several authors. For a list of references see [2]. Ling [11] has specifically investigated the associative property (A). Bisymmetry (B) has been used by Aczél [1] and Fodor and Marichal [7] to characterize certain mean values. This property expresses that aggregation can be performed first on the rows, then on the columns of any square matrix, or conversely. The next proposition presents an immediate link between (B) and (AD).

Proposition 3.4 For every aggregation function $M^{(2)}$ defined on $\mathbb{I}^{2}$, we have $(I, B) \Rightarrow(A D)$.

The next algebraic properties concern aggregators.

Definition 3.6 The aggregator $M$ defined on $\bigcup_{m \geq 1} \mathbb{I}^{m}$ is 
- associative (A) if each subset of consecutive elements from $\left(x_{1}, \ldots, x_{m}\right)$ can be substituted by the partial aggregation of this subset without changing the global aggregation, i.e. formally, if $M^{(1)}(x)=x \forall x \in \mathbb{I}$ and if, for all $m \in \mathbb{N}^{*}$, all $\left(x_{1}, \ldots, x_{m}\right) \in \mathbb{I}^{m}$ and all $0 \leq j<k \leq m$, we have

$$
\begin{aligned}
& M^{(m)}\left(x_{1}, \ldots, x_{j}, x_{j+1}, \ldots, x_{k}, x_{k+1}, \ldots, x_{m}\right) \\
= & M^{(m-k+j+1)}\left(x_{1}, \ldots, x_{j}, M^{(k-j)}\left(x_{j+1}, \ldots, x_{k}\right), x_{k+1}, \ldots, x_{m}\right) .
\end{aligned}
$$

- decomposable (D) if each element of any subset of consecutive elements from $\left(x_{1}, \ldots, x_{m}\right)$ can be substituted by the partial aggregation of this subset without changing the global aggregation, i.e. formally, if $M^{(1)}(x)=x \forall x \in \mathbb{I}$ and if, for all $m \in \mathbb{N}^{*}$, all $\left(x_{1}, \ldots, x_{m}\right) \in \mathbb{I}^{m}$ and all $0 \leq j<k \leq m$, we have

$$
\begin{aligned}
& M^{(m)}\left(x_{1}, \ldots, x_{j}, x_{j+1}, \ldots, x_{k}, x_{k+1}, \ldots, x_{m}\right) \\
= & M^{(m)}\left(x_{1}, \ldots, x_{j},(k-j) \cdot M^{(k-j)}\left(x_{j+1}, \ldots, x_{k}\right), x_{k+1}, \ldots, x_{m}\right) .
\end{aligned}
$$

- strongly decomposable (SD) if each element of any subset of elements from $\left(x_{1}, \ldots, x_{m}\right)$ can be substituted by the partial aggregation of this subset without changing the global aggregation, i.e. formally, if $M^{(1)}(x)=x \forall x \in \mathbb{I}$ and if, for all $m \in \mathbb{N}^{*}$, all $\left(x_{1}, \ldots, x_{m}\right) \in \mathbb{I}^{m}$ and all $N=\left\{i_{1}, \ldots, i_{p}\right\} \subseteq N_{m}$, with $i_{1}<\ldots<i_{p}$, we have

$$
M^{(m)}\left(x_{1}, \ldots, x_{m}\right)=M^{(m)}\left(x_{1}^{\prime}, \ldots, x_{m}^{\prime}\right)
$$

where, for all $i \in N_{m}$,

$$
x_{i}^{\prime}= \begin{cases}x_{i} & \text { if } i \notin N, \\ M^{(p)}\left(x_{i_{1}}, \ldots, x_{i_{p}}\right) & \text { otherwise. }\end{cases}
$$

- strongly bisymmetric (SB) if $M^{(1)}(x)=x \forall x \in \mathbb{I}$ and if, for all $m, p \in \mathbb{N}^{*}$,

$$
\begin{aligned}
& M^{(p)}\left(M^{(m)}\left(x_{11}, \ldots, x_{1 m}\right), \ldots, M^{(m)}\left(x_{p 1}, \ldots, x_{p m}\right)\right) \\
= & M^{(m)}\left(M^{(p)}\left(x_{11}, \ldots, x_{p 1}\right), \ldots, M^{(p)}\left(x_{1 m}, \ldots, x_{p m}\right)\right)
\end{aligned}
$$

for all matrices

$$
X=\left(\begin{array}{ccc}
x_{11} & \cdots & x_{1 m} \\
\vdots & & \vdots \\
x_{p 1} & \cdots & x_{p m}
\end{array}\right) \in \mathbb{I}^{p \times m} .
$$

Note that those definitions make sense only if, for all $m \in \mathbb{N}^{*}, M^{(m)}$ takes its values in $\mathbb{I}$.

Associativity (A) is a well-known algebraic property which allows to omit "parentheses" in an aggregation of at least three elements (see e.g. [2]). Observe that, if the aggregator $M$ is associative, then the function $M^{(2)}$ is associative (just set $m=3$ in Definition 3.6). Of course, associativity can be viewed as an iterative property since it allows to define completely any aggregator $M$ only from its function $M^{(2)}$.

Decomposability is a property introduced by Kolmogoroff [10] and Nagumo [14] in the case of symmetric aggregators (Sy)(see also [7]). In the nonsymmetric case, we generalize this property in two ways: decomposability (D) and strong decomposability (SD). Of course, under (Sy), these two properties are identical. We also introduce the property of strong bisymmetry (SB) as a generalization of $(\mathrm{B})$.

The next proposition points out a link between (A) and (D). 
Proposition 3.5 For every aggregator $M$ defined on $\bigcup_{m \geq 1} \mathbb{I}^{m}$, we have $(I, A) \Rightarrow(D)$.

Proof. Let $m \in \mathbb{N}^{*}$ and $\left(x_{1}, \ldots, x_{m}\right) \in \mathbb{I}^{m}$. We have, if $0 \leq j<k \leq m$,

$$
\begin{array}{ll} 
& M^{(m)}\left(x_{1}, \ldots, x_{j}, x_{j+1}, \ldots, x_{k}, x_{k+1}, \ldots, x_{m}\right) \\
\stackrel{(A)}{=} & M^{(m-k+j+1)}\left(x_{1}, \ldots, x_{j}, M^{(k-j)}\left(x_{j+1}, \ldots, x_{k}\right), x_{k+1}, \ldots, x_{m}\right) \\
\stackrel{(I)}{=} & M^{(m-k+j+1)}\left(x_{1}, \ldots, x_{j}, M^{(k-j)}\left((k-j) \cdot M^{(k-j)}\left(x_{j+1}, \ldots, x_{k}\right)\right), x_{k+1}, \ldots, x_{m}\right) \\
\stackrel{(A)}{=} & M^{(m)}\left(x_{1}, \ldots, x_{j},(k-j) \cdot M^{(k-j)}\left(x_{j+1}, \ldots, x_{k}\right), x_{k+1}, \ldots, x_{m}\right) .
\end{array}
$$

\section{Characterization of some aggregation functions}

This section is devoted to aggregation functions $M^{(m)}$ which are increasing and stable for positive linear transformations. The next definition introduces some of them (all are defined on $\mathbb{I}^{m}$ ).

Definition 4.1 Let $m \in \mathbb{N}^{*}$.

- For any weight vector $\omega^{(m)}=\left(\omega_{1}^{(m)}, \ldots, \omega_{m}^{(m)}\right) \in \mathbb{I}^{m}$ such that

$$
\sum_{i=1}^{m} \omega_{i}^{(m)}=1
$$

the weighted arithmetic mean function $W A M_{\omega^{(m)}}^{(m)}$ and the ordered weighted averaging function $O W A_{\omega^{(m)}}^{(m)}$ associated to $\omega^{(m)}$, are respectively defined by

$$
\begin{aligned}
W A M_{\omega^{(m)}}^{(m)}\left(x_{1}, \ldots, x_{m}\right) & =\sum_{i=1}^{m} \omega_{i}^{(m)} x_{i} \quad \forall\left(x_{1}, \ldots, x_{m}\right) \in \mathbb{I}^{m}, \\
O W A_{\omega^{(m)}}^{(m)}\left(x_{1}, \ldots, x_{m}\right) & =\sum_{i=1}^{m} \omega_{i}^{(m)} x_{(i)} \quad \forall\left(x_{1}, \ldots, x_{m}\right) \in \mathbb{I}^{m} .
\end{aligned}
$$

- The arithmetic mean function $A M^{(m)}$ is defined by

$$
A M^{(m)}\left(x_{1}, \ldots, x_{m}\right)=\frac{1}{m} \sum_{i=1}^{m} x_{i} \forall\left(x_{1}, \ldots, x_{m}\right) \in \mathbb{I}^{m} .
$$

- For any $i \in N_{m}$, the projection function $P_{i}^{(m)}$ associated to the ith argument is defined by

$$
P_{i}^{(m)}\left(x_{1}, \ldots, x_{m}\right)=x_{i} \quad \forall\left(x_{1}, \ldots, x_{m}\right) \in \mathbb{I}^{m} .
$$

- The minimum function $M I N^{(m)}$ and the maximum function $M A X^{(m)}$ are respectively defined by

$$
\begin{aligned}
M I N^{(m)}\left(x_{1}, \ldots, x_{m}\right) & =\min _{i \in N_{m}} x_{i} \forall\left(x_{1}, \ldots, x_{m}\right) \in \mathbb{I}^{m}, \\
M A X^{(m)}\left(x_{1}, \ldots, x_{m}\right) & =\max _{i \in N_{m}} x_{i} \quad \forall\left(x_{1}, \ldots, x_{m}\right) \in \mathbb{I}^{m} .
\end{aligned}
$$




\begin{tabular}{|l|l|}
\hline$(\theta, \bar{\theta})$ & $M^{(2)}$ \\
\hline \hline$(\theta, \bar{\theta})=(0,0)$ & $\mathrm{MIN}^{(2)}$ \\
$(\theta, \bar{\theta})=(1,1)$ & $\operatorname{MAX}^{(2)}$ \\
$(\theta, \bar{\theta})=(0,1)$ & $\mathrm{P}_{1}^{(2)}$ \\
$(\theta, \bar{\theta})=(1,0)$ & $\mathrm{P}_{2}^{(2)}$ \\
$\theta+\bar{\theta}=1$ & $\operatorname{WAM}_{(1-\theta, \theta)}^{(2)}$ \\
$\theta=\bar{\theta}$ & $\operatorname{OWA}_{(1-\theta, \theta)}^{(2)}$ \\
\hline
\end{tabular}

Table 1: Some examples

- For any nonempty subset $N^{(m)} \subseteq N_{m}$, the partial minimum function $\operatorname{MIN}_{N^{(m)}}^{(m)}$ and the partial maximum function $M A X_{N^{(m)}}^{(m)}$ associated to $N^{(m)}$, are respectively defined by

$$
\begin{aligned}
\operatorname{MIN}_{N^{(m)}}^{(m)}\left(x_{1}, \ldots, x_{m}\right) & =\min _{i \in N^{(m)}} x_{i} \forall\left(x_{1}, \ldots, x_{m}\right) \in \mathbb{I}^{m}, \\
M A X_{N^{(m)}}^{(m)}\left(x_{1}, \ldots, x_{m}\right) & =\max _{i \in N^{(m)}} x_{i} \forall\left(x_{1}, \ldots, x_{m}\right) \in \mathbb{I}^{m} .
\end{aligned}
$$

The following result shows that any aggregation function $M^{(2)}$ defined on $\mathbb{I}^{2}$ and fulfilling (In, SPL) is completely defined by the values $M^{(2)}(0,1)$ and $M^{(2)}(1,0)$.

Proposition 4.1 The aggregation function $M^{(2)}$ defined on $\mathbb{I}^{2}$ fulfils (In, SPL) if and only if, for all $\left(x_{1}, x_{2}\right) \in \mathbb{I}^{2}$, we have

$$
\begin{aligned}
M^{(2)}\left(x_{1}, x_{2}\right) & = \begin{cases}(1-\theta) x_{1}+\theta x_{2} & \text { if } x_{1} \leq x_{2} \\
\bar{\theta} x_{1}+(1-\bar{\theta}) x_{2} & \text { if } x_{1} \geq x_{2}\end{cases} \\
& =\bar{\theta} x_{1}+\theta x_{2}+(1-\theta-\bar{\theta})\left(x_{1} \wedge x_{2}\right)
\end{aligned}
$$

with $\theta, \bar{\theta} \in$ II. Moreover, we have $\theta=M^{(2)}(0,1)$ and $\bar{\theta}=M^{(2)}(1,0)$.

Proof. (Sufficiency). Easy.

(Necessity). Let $\left(x_{1}, x_{2}\right) \in \mathbb{I}^{2}$. If $x_{1} \leq x_{2}$ then we have

$$
M^{(2)}\left(x_{1}, x_{2}\right) \stackrel{(S P L)}{=}\left(x_{2}-x_{1}\right) M^{(2)}(0,1)+x_{1}=(1-\theta) x_{1}+\theta x_{2}
$$

with $\theta=M^{(2)}(0,1)$. Moreover, $\theta \in \mathbb{I}$ since, by Propositions 3.1 and $3.2, M^{(2)}$ is compensative.

One proceeds similarly if $x_{1} \geq x_{2}$.

Some particular examples according to the values of $\theta$ and $\bar{\theta}$ can be found in Table 1 . Moreover, the next corollary trivially follows.

Corollary 4.1 The aggregation function $M^{(2)}$ defined on $\mathbb{I}^{2}$ fulfils (Sy, In, SPL) if and only if there exists $\omega^{(2)} \in \mathbb{I}^{2}$ such that

$$
M^{(2)}=O W A_{\omega^{(2)}}^{(2)}
$$

Note that a complete characterization of the $\mathrm{OWA}_{\omega(m)}^{(m)}$ functions can be found in [12] (see also [9, p.133]).

The next two theorems describe the families of aggregation functions $M^{(2)}$ defined on $\mathbb{I}^{2}$ and fulfilling (In, SPL, A), (In, SPL, AD) and (In, SPL, B) respectively.

Theorem 4.1 The aggregation function $M^{(2)}$ defined on $\mathbb{I}^{2}$ fulfils (In, SPL, A) if and only if

$$
M^{(2)} \in\left\{M I N^{(2)}, M A X^{(2)}, P_{1}^{(2)}, P_{2}^{(2)}\right\} .
$$


Proof. (Sufficiency). Trivial.

(Necessity). Set $\theta=M^{(2)}(0,1)$ and $\bar{\theta}=M^{(2)}(1,0)$. By Proposition 4.1, we only have to prove that $\theta, \bar{\theta} \in\{0,1\}$. The aggregation function $M^{(2)}$ must fulfil the equation

$$
M^{(2)}\left(M^{(2)}\left(x_{1}, x_{2}\right), x_{3}\right)=M^{(2)}\left(x_{1}, M^{(2)}\left(x_{2}, x_{3}\right)\right)
$$

for all $\left(x_{1}, x_{2}, x_{3}\right) \in \mathbb{I}^{3}$. In particular, for $\left(x_{1}, x_{2}, x_{3}\right)=e_{3}^{(3)}$, we obtain, by (SSI), $\theta \in\{0,1\}$. Also, for $\left(x_{1}, x_{2}, x_{3}\right)=e_{1}^{(3)}$, we obtain $\bar{\theta} \in\{0,1\}$.

Theorem 4.2 Let $M^{(2)}$ be any aggregation function defined on $\mathbb{I}^{2}$. Then the following three assertions are equivalent:

$$
\begin{aligned}
\text { (i) } & M^{(2)} \text { fulfils (In, SPL, AD), } \\
\text { (ii) } & M^{(2)} \text { fulfils (In, SPL, B), } \\
\text { (iii) } & M^{(2)} \in\left\{M I N^{(2)}, M A X^{(2)}\right\} \cup\left\{W A M_{\omega^{(2)}}^{(2)} \mid \omega^{(2)} \in \mathbb{I}^{2}\right\} .
\end{aligned}
$$

Proof. $($ iii $) \Rightarrow($ ii $)$. Trivial.

$($ ii $) \Rightarrow(i)$. It is a straightforward consequence of Propositions 3.2 and 3.4.

(i) $\Rightarrow($ iii $)$. Set $\theta=M^{(2)}(0,1)$ and $\bar{\theta}=M^{(2)}(1,0)$. By Proposition 4.1 , we only have to prove that $(\theta, \bar{\theta}) \in\{(0,0),(1,1)\}$ or $\theta+\bar{\theta}=1$. The aggregation function $M^{(2)}$ must fulfil the equation

$$
M^{(2)}\left(x_{1}, M^{(2)}\left(x_{2}, x_{3}\right)\right)=M^{(2)}\left(M^{(2)}\left(x_{1}, x_{2}\right), M^{(2)}\left(x_{1}, x_{3}\right)\right)
$$

for all $\left(x_{1}, x_{2}, x_{3}\right) \in \mathbb{I}^{3}$. In particular, for $\left(x_{1}, x_{2}, x_{3}\right)=(\theta, 0,1)$, we obtain, by Proposition 4.1,

$$
\theta=M^{(2)}(\theta \bar{\theta}, \theta(2-\theta))=(1-\theta) \theta \bar{\theta}+\theta^{2}(2-\theta)
$$

and so, $\theta=0$ or $\theta=1$ or $\theta+\bar{\theta}=1$. Similarly, for $\left(x_{1}, x_{2}, x_{3}\right)=(\bar{\theta}, 1,0)$, we obtain, by Proposition 4.1 ,

$$
\bar{\theta}=M^{(2)}\left((1-\theta) \bar{\theta}+\theta, \bar{\theta}^{2}\right)=\bar{\theta}^{2}(1-\theta)+\bar{\theta} \theta+(1-\bar{\theta}) \bar{\theta}^{2}
$$

and so, $\bar{\theta}=0$ or $\bar{\theta}=1$ or $\theta+\bar{\theta}=1$.

Corollary 4.2 Let $M^{(2)}$ be any aggregation function defined on $\mathbb{I}^{2}$. Then the following three assertions are equivalent:

(i) $M^{(2)}$ fulfils (Sy, In, SPL, AD),

(ii) $M^{(2)}$ fulfils $(S y, I n, S P L, B)$,

(iii) $M^{(2)} \in\left\{M I N^{(2)}, M A X^{(2)}, A M^{(2)}\right\}$.

Before obtaining the description of the family of functions $M^{(m)}$ defined on $\mathbb{I}^{m}(m \geq 2)$ and fulfilling (In, SPL, B), we need three technical lemmas.

Lemma 4.1 Let $m \in \mathbb{N}^{*}, m \geq 2$. If the aggregation function $M^{(m)}$ defined on $\mathbb{I}^{m}$ fulfils (In, $S P L, B)$ and if there exists $N \subseteq N_{m}$ such that $\theta_{N}^{(m)} \in(0,1)$ then $M^{(m)}$ is additive, i.e.

$$
M^{(m)}\left(u_{1}+v_{1}, \ldots, u_{m}+v_{m}\right)=M^{(m)}\left(u_{1}, \ldots, u_{m}\right)+M^{(m)}\left(v_{1}, \ldots, v_{m}\right)
$$

for all $\left(u_{1}, \ldots, u_{m}\right),\left(v_{1}, \ldots, v_{m}\right) \in \mathbb{I}^{m}$ with $u_{i}+v_{i} \in \mathbb{I} \forall i \in N_{m}$. 
Proof. Let $\left(u_{1}, \ldots, u_{m}\right),\left(v_{1}, \ldots, v_{m}\right) \in \mathbb{I}^{m}$ with $u_{i}+v_{i} \in \mathbb{I} \forall i \in N_{m}$ and $\operatorname{set} \alpha=\inf \left\{\theta_{N}^{(m)}, 1-\right.$ $\left.\theta_{N}^{(m)}\right\} \in(0,1)$.

(i) Assume first that $u_{i}, v_{i} \leq \alpha \forall i \in N_{m}$. Consider the square matrix $X$ of $m$ rows $r_{i}$ and $m$ columns $c_{j}\left(i, j \in N_{m}\right)$, where $r_{i}$ is defined as follows (for $i \in N_{m}$ ):

$$
r_{i}=\frac{1}{2}\left(\frac{u_{i}}{\theta_{N}^{(m)}}+1\right) e_{N}^{(m)}+\frac{1}{2} \frac{v_{i}}{1-\theta_{N}^{(m)}} \bar{e}_{N}^{(m)} .
$$

On the one hand, by (SPL), we have, for all $i \in N_{m}$ :

$$
M^{(m)}\left(r_{i}\right)=\frac{1}{2} \frac{v_{i}}{1-\theta_{N}^{(m)}}+\frac{1}{2}\left(\frac{u_{i}}{\theta_{N}^{(m)}}+1-\frac{v_{i}}{1-\theta_{N}^{(m)}}\right) \theta_{N}^{(m)}=\frac{u_{i}+v_{i}}{2}+\frac{\theta_{N}^{(m)}}{2}
$$

and thus

$$
M^{(m)}\left(M^{(m)}\left(r_{1}\right), \ldots, M^{(m)}\left(r_{m}\right)\right)=\frac{1}{2} M^{(m)}\left(u_{1}+v_{1}, \ldots, u_{m}+v_{m}\right)+\frac{\theta_{N}^{(m)}}{2} .
$$

On the other hand, for all $j \in N_{m}$, we have

$$
M^{(m)}\left(c_{j}\right)= \begin{cases}\frac{1}{2}\left(\frac{M^{(m)}\left(u_{1}, \ldots, u_{m}\right)}{\theta_{N}^{(m)}}+1\right) & \text { if } j \in N \\ \frac{1}{2} \frac{M^{(m)}\left(v_{1}, \ldots, v_{m}\right)}{1-\theta_{N}^{(m)}} & \text { otherwise. }\end{cases}
$$

However, by Propositions 3.1 and 3.2, $M^{(m)}$ fulfils (Comp). Hence, by (SPL), we have,

$$
\begin{aligned}
& M^{(m)}\left(M^{(m)}\left(c_{1}\right), \ldots, M^{(m)}\left(c_{m}\right)\right) \\
= & M^{(m)}\left[\frac{1}{2}\left(\frac{M^{(m)}\left(u_{1}, \ldots, u_{m}\right)}{\theta_{N}^{(m)}}+1\right) e_{N}^{(m)}+\frac{1}{2} \frac{M^{(m)}\left(v_{1}, \ldots, v_{m}\right)}{1-\theta_{N}^{(m)}} \bar{e}_{N}^{(m)}\right] \\
= & \frac{1}{2}\left[M^{(m)}\left(u_{1}, \ldots, u_{m}\right)+M^{(m)}\left(v_{1}, \ldots, v_{m}\right)\right]+\frac{\theta_{N}^{(m)}}{2} .
\end{aligned}
$$

Since $M^{(m)}$ fulfils (B), we have

$$
M^{(m)}\left(u_{1}+v_{1}, \ldots, u_{m}+v_{m}\right)=M^{(m)}\left(u_{1}, \ldots, u_{m}\right)+M^{(m)}\left(v_{1}, \ldots, v_{m}\right) .
$$

(ii) In the general case, we have,

$$
\begin{aligned}
& M^{(m)}\left(u_{1}+v_{1}, \ldots, u_{m}+v_{m}\right) \stackrel{(S P L)}{=} \frac{1}{\alpha} M^{(m)}\left(\alpha u_{1}+\alpha v_{1}, \ldots, \alpha u_{m}+\alpha v_{m}\right) \\
& \stackrel{(i)}{=} \quad \frac{1}{\alpha} M^{(m)}\left(\alpha u_{1}, \ldots, \alpha u_{m}\right)+\frac{1}{\alpha} M^{(m)}\left(\alpha v_{1}, \ldots, \alpha v_{m}\right) \\
& \stackrel{(S P L)}{=} \quad M^{(m)}\left(u_{1}, \ldots, u_{m}\right)+M^{(m)}\left(v_{1}, \ldots, v_{m}\right) \text {. }
\end{aligned}
$$

Lemma 4.2 Let $m \in \mathbb{N}^{*}, m \geq 2$. If the aggregation function $M^{(m)}$ defined on $\mathbb{I}^{m}$ fulfils (In, $S P L, B)$, and if $\theta_{N}^{(m)} \in\{0,1\} \forall N \subseteq N_{m}$, then, setting $N_{\max }=\left\{i \in N_{m} \mid \theta_{i}^{(m)}=1\right\}$ and assuming $N_{\max } \neq \emptyset$, we have, for all $N \subseteq N_{m}$ :

$$
N \cap N_{\max }=\emptyset \Rightarrow \theta_{N}^{(m)}=0
$$


Proof. The result is trivial if $m=2$. Otherwise, use induction on $|N|$.

The result holds for $|N| \in\{0,1\}$. Assume that it holds for $|N|=n \geq 1$ and show that it holds for $|N|=n+1$. Assume that $N \cap N_{\max }=\emptyset$ and consider the square matrix $X$ of $m$ rows $r_{i}$ and $m$ columns $c_{j}\left(i, j \in N_{m}\right)$, where $r_{i}$ is defined as follows (for $i \in N_{m}$ ):

$$
r_{i}= \begin{cases}(m \cdot 0) & \text { if } i \notin N \\ e_{N}^{(m)} & \text { if } i=q \\ (m \cdot 1) & \text { if } i \in N \backslash\{q\}\end{cases}
$$

where $q \in N$. On the one hand, for all $i \in N \backslash\{q\}$, we have $M^{(m)}\left(r_{i}\right)=1$. On the other hand, for all $j \in N_{m}$, we have

$$
M^{(m)}\left(c_{j}\right)= \begin{cases}0 & \text { if } j \notin N \text { (by induction) }, \\ \theta_{N}^{(m)} & \text { if } j \in N .\end{cases}
$$

Indeed, if $j \notin N$, we have $c_{j}=e_{N \backslash\{q\}}^{(m)}$ and $(N \backslash\{q\}) \cap N_{\max }=\emptyset$.

Now, let $j_{0} \in N \backslash\{q\}$. In particular, we have $j_{0} \notin N_{\max }$. Consider the square matrix $X^{\prime}$ of $m$ rows $r_{i}^{\prime}$ and $m$ columns $c_{j}^{\prime}\left(i, j \in N_{m}\right)$, where $r_{i}^{\prime}=r_{i}$ for all $i \in N_{m} \backslash\left\{j_{0}\right\}$ and $r_{j_{0}}^{\prime}=\bar{e}_{j_{0}}^{(m)}$. We then have

$$
M^{(m)}\left(\bar{e}_{j_{0}}^{(m)}\right)=\bar{\theta}_{j_{0}}^{(m)}=1
$$

since, by (In), $\bar{\theta}_{j_{0}}^{(m)} \geq \theta_{i}^{(m)}$ for all $i \in N_{\max }$. So we have

$$
M^{(m)}\left(M^{(m)}\left(r_{1}^{\prime}\right), \ldots, M^{(m)}\left(r_{m}^{\prime}\right)\right)=M^{(m)}\left(M^{(m)}\left(r_{1}\right), \ldots, M^{(m)}\left(r_{m}\right)\right) .
$$

However, since $c_{j_{0}}^{\prime}=e_{N \backslash\left\{j_{0}\right\}}^{(m)}$, we have $M^{(m)}\left(c_{j_{0}}^{\prime}\right)=0$ (by induction) and

$$
M^{(m)}\left(c_{j}^{\prime}\right)= \begin{cases}0 & \text { if } j \notin N, \\ 0 & \text { if } j=j_{0}, \\ \theta_{N}^{(m)} & \text { if } j \in N \backslash\left\{j_{0}\right\} .\end{cases}
$$

Consequently, since $M^{(m)}$ fulfils (SPL) and (B), we have

$$
\begin{aligned}
{\left[\theta_{N}^{(m)}\right]^{2} } & =M^{(m)}\left(M^{(m)}\left(c_{1}\right), \ldots, M^{(m)}\left(c_{m}\right)\right)=M^{(m)}\left(M^{(m)}\left(r_{1}\right), \ldots, M^{(m)}\left(r_{m}\right)\right) \\
& =M^{(m)}\left(M^{(m)}\left(r_{1}^{\prime}\right), \ldots, M^{(m)}\left(r_{m}^{\prime}\right)\right)=M^{(m)}\left(M^{(m)}\left(c_{1}^{\prime}\right), \ldots, M^{(m)}\left(c_{m}^{\prime}\right)\right) \\
& =\theta_{N}^{(m)} M^{(m)}\left(e_{N \backslash\left\{j_{0}\right\}}^{(m)}\right)=0 .
\end{aligned}
$$

Lemma 4.3 Let $m \in \mathbb{N}^{*}, m \geq 2$. If the aggregation function $M^{(m)}$ defined on $\mathbb{I}^{m}$ fulfils (In, SPL, $B)$, if $\theta_{N}^{(m)} \in\{0,1\} \forall N \subseteq N_{m}$ and $\theta_{i}^{(m)}=0 \forall i \in N_{m}$, then, setting $N_{\min }=\left\{i \in N_{m} \mid \bar{\theta}_{i}^{(m)}=0\right\}$, we have $N_{\min } \neq \emptyset$ and $\theta_{N_{\min }}^{(m)}=1$.

Proof. If $m=2$ then $\bar{\theta}_{1}^{(2)}=\theta_{2}^{(2)}=0$ and $\bar{\theta}_{2}^{(2)}=\theta_{1}^{(2)}=0$. Otherwise, let $N_{\text {min }}^{*} \subseteq N_{m}$ with a minimal cardinality such that $\theta_{N_{\text {min }}^{*}}^{(m)}=1$. The existence of such a $N_{\min }^{*}$ is trivial since $\theta_{N_{m}}^{(m)}=1$, and we clearly have $\left|N_{\min }^{*}\right| \geq 2$. Let us prove that $N_{\text {min }}^{*} \subseteq N_{\text {min }}$. Assume that there exists $k \in N_{\text {min }}^{*}$ such that $\bar{\theta}_{k}^{(m)}=1$. Consider the square matrix $X$ of $m$ rows $r_{i}$ and $m$ columns $c_{j}\left(i, j \in N_{m}\right)$, where $r_{i}$ is defined as follows (for $i \in N_{m}$ ):

$$
r_{i}= \begin{cases}(m \cdot 0) & \text { if } i \notin N_{\min }^{*} \\ e_{N_{\min }^{*}}^{(m)} & \text { if } i=k \\ \bar{e}_{k}^{(m)} & \text { if } i \in N_{\min }^{*} \backslash\{k\}\end{cases}
$$


On the one hand, we have, for all $i \in N_{m}$ :

$$
M^{(m)}\left(r_{i}\right)= \begin{cases}0 & \text { if } i \notin N_{\min }^{*} \\ 1 & \text { if } i \in N_{\min }^{*}\end{cases}
$$

and thus

$$
M^{(m)}\left(M^{(m)}\left(r_{1}\right), \ldots, M^{(m)}\left(r_{m}\right)\right)=M^{(m)}\left(e_{N_{\min }^{*}}^{(m)}\right)=\theta_{N_{\min }^{*}}^{(m)}=1 .
$$

On the other hand, for all $j \in N_{m}$, we have

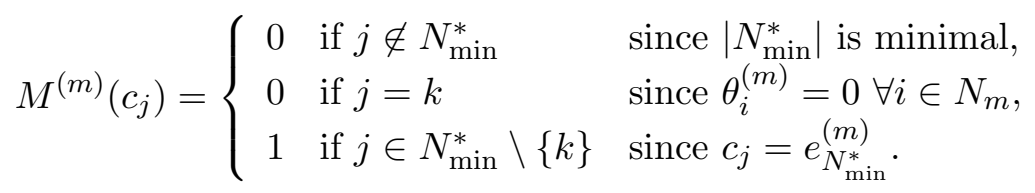

Since $M^{(m)}$ fulfils (B), we have

$$
1=M^{(m)}\left(M^{(m)}\left(c_{1}\right), \ldots, M^{(m)}\left(c_{m}\right)\right)=M^{(m)}\left(e_{N_{\min }^{*} \backslash\{k\}}^{(m)}\right),
$$

a contradiction since $\left|N_{\text {min }}^{*}\right|$ is minimal.

Now, let us prove that $N_{\min } \subseteq N_{\min }^{*}$. For all $i \notin N_{\min }^{*}$, we have, by (In), $\bar{\theta}_{i}^{(m)} \geq \theta_{N_{\min }^{*}}^{(m)}=1$, that is $i \notin N_{\min }$.

Theorem 4.3 Let $m \in \mathbb{N}^{*}, m \geq 2$. The aggregation function $M^{(m)}$ defined on $\mathbb{I}^{m}$ fulfils (In, $S P L, B)$ if and only if

$$
M^{(m)} \in\left\{M I N_{N^{(m)}}^{(m)}, M A X_{N^{(m)}}^{(m)} \mid N^{(m)} \subseteq N_{m}\right\} \cup\left\{W A M_{\omega^{(m)}}^{(m)} \mid \omega^{(m)} \in \mathbb{I}^{m}\right\} .
$$

Proof. (Sufficiency). Trivial.

(Necessity). Let $\left(x_{1}, \ldots, x_{m}\right) \in \mathbb{I}^{m}$. The values $\theta_{N}^{(m)}\left(N \subseteq N_{m}\right)$ fulfil the assumptions of exactly one of Lemmas 4.1, 4.2 or 4.3. We then have three exclusive cases:

(i) Under the assumptions of Lemma 4.1, we have, by (SPL),

$$
M^{(m)}\left(x_{1}, \ldots, x_{m}\right)=\sum_{i=1}^{m} \theta_{i}^{(m)} x_{i}
$$

with, by $(\mathrm{I}), \sum_{i} \theta_{i}^{(m)}=M^{(m)}(m \cdot 1)=1$.

(ii) Under the assumptions of Lemma 4.2, there exists $p \in N_{\max }$ such that $x_{p}=\max _{i \in N_{\max }} x_{i}$ and we have

$$
M^{(m)}\left(x_{1}, \ldots, x_{m}\right) \stackrel{(I n)}{\leq} M^{(m)}\left(x_{p} e_{N_{\max }}^{(m)}+\bar{e}_{N_{\max }}^{(m)}\right) \stackrel{(S P L)}{=} x_{p}+\left(1-x_{p}\right) \bar{\theta}_{N_{\max }}^{(m)}=x_{p}
$$

and

$$
M^{(m)}\left(x_{1}, \ldots, x_{m}\right) \stackrel{(I n)}{\geq} M^{(m)}\left(x_{p} e_{p}^{(m)}\right) \stackrel{(S P L)}{=} x_{p} \theta_{p}^{(m)}=x_{p} .
$$

Therefore, we have

$$
M^{(m)}\left(x_{1}, \ldots, x_{m}\right)=\max _{i \in N_{\max }} x_{i} .
$$

(iii) Under the assumptions of Lemma 4.3, there exists $p \in N_{\min }$ such that $x_{p}=\min _{i \in N_{\min }} x_{i}$ and we have, since $p \in N_{\min }$ :

$$
M^{(m)}\left(x_{1}, \ldots, x_{m}\right) \stackrel{(I n)}{\leq} M^{(m)}\left(x_{p} e_{p}^{(m)}+\bar{e}_{p}^{(m)}\right) \stackrel{(S P L)}{=} x_{p}+\left(1-x_{p}\right) \bar{\theta}_{p}^{(m)}=x_{p}
$$




\begin{tabular}{|l|l|l|}
\hline In, SPL, A $(m=2)$ & Sy, In, SPL, A $(m=2)$ & In, SSI, SSN, A $(m=2)$ \\
\hline $\operatorname{MIN}^{(2)}, \mathrm{MAX}^{(2)}, \mathrm{P}_{1}^{(2)}, \mathrm{P}_{2}^{(2)}$. & $\mathrm{MIN}^{(2)}, \mathrm{MAX}^{(2)}$. & $\mathrm{P}_{1}^{(2)}, \mathrm{P}_{2}^{(2)}$. \\
\hline \hline In, SPL, AD $(m=2)$ & Sy, In, SPL, AD $(m=2)$ & In, SSI, SSN, AD $(m=2)$ \\
\hline $\mathrm{MIN}^{(2)}, \mathrm{MAX}^{(2)},\left\{\mathrm{WAM}_{\omega^{(2)}}^{(2)} \mid \omega^{(2)} \in \mathbb{I}^{2}\right\}$. & $\mathrm{MIN}^{(2)}, \mathrm{MAX}^{(2)}, \mathrm{AM}^{(2)}$. & $\left\{\mathrm{WAM}_{\omega^{(2)}}^{(2)} \mid \omega^{(2)} \in \mathbb{I}^{2}\right\}$. \\
\hline \hline In, SPL, B $(m \geq 2)$ & Sy, In, SPL, B $(m \geq 2)$ & In, SSI, SSN, B $(m \geq 2)$ \\
\hline $\begin{array}{l}\left\{\mathrm{MIN}_{N^{(m)}}^{(m)}, \mathrm{MAX}_{N^{(m)}}^{(m)} \mid N^{(m)} \subseteq N_{m}\right\}, \\
\left\{\mathrm{WAM}_{\omega(m)}^{(m)} \mid \omega^{(m)} \in \mathbb{I}^{m}\right\} .\end{array}$ & $\mathrm{MIN}^{(m)}, \mathrm{MAX}^{(m)}, \mathrm{AM}^{(m)}$. & $\left\{\mathrm{WAM}_{\omega^{(m)}}^{(m)} \mid \omega^{(m)} \in \mathbb{I}^{m}\right\}$. \\
\end{tabular}

Table 2: Results from Section 4

and

$$
M^{(m)}\left(x_{1}, \ldots, x_{m}\right) \stackrel{(I n)}{\geq} M^{(m)}\left(x_{p} e_{N_{\min }}^{(m)} \stackrel{(S P L)}{=} x_{p} \theta_{N_{\min }}^{(m)}=x_{p} .\right.
$$

Therefore, we have

$$
M^{(m)}\left(x_{1}, \ldots, x_{m}\right)=\min _{i \in N_{\min }} x_{i}
$$

To summarize this section, we present a table containing the characterizations obtained above (Table 2). The table also contains some corollaries which can be checked easily (recall that (SSI, SSN) implies (SPL)). Among them, we can find a complete characterization of the weighted arithmetic mean functions with $m$ arguments. Note that another characterization of this family can be found in [2, pp.234-239] (see also [5]). Moreover, by introducing new properties, we can obtain other corollaries. For instance, $\mathrm{AM}^{(m)}$ alone could be characterized using a property of strict increasing monotonicity.

\section{Characterization of some aggregators}

This section is devoted to aggregators $M$ which are increasing and stable for positive linear transformations. The next definition introduces some of them (all are defined on $\bigcup_{m \geq 1} \mathbb{I}^{m}$ ).

\section{Definition 5.1}

- For any sequence $\omega=\left(\omega^{(m)}\right)_{m \in \mathbb{N}^{*}}$ of weight vectors $\omega^{(m)} \in \mathbb{I}^{m}$, such that

$$
\sum_{i=1}^{m} \omega_{i}^{(m)}=1 \forall m \in \mathbb{N}^{*}
$$

the weighted arithmetic mean aggregator $W A M_{\omega}$ associated to $\omega$ is the aggregator $\left(\operatorname{WAM}_{\omega(m)}^{(m)}\right)_{m \in \mathbb{N}^{*}}$.

- For any $\theta \in \mathbb{I}$, the decomposable weighted arithmetic mean aggregator DWAM $M_{\theta}$ associated to $\theta$ is the aggregator $\left(W A M_{\omega(m)}^{(m)}\right)_{m \in \mathbb{N}^{*}}$ where, for all $i \in N_{m}$,

$$
\omega_{i}^{(m)}=\frac{(1-\theta)^{m-i} \theta^{i-1}}{\sum_{j=1}^{m}(1-\theta)^{m-j} \theta^{j-1}} .
$$

- The arithmetic mean aggregator $A M$ is the aggregator $\left(A M^{(m)}\right)_{m \in \mathbb{N}^{*}}$. 
- The first projection aggregator FP and the last projection aggregator LP are the aggregators $\left(P_{1}^{(m)}\right)_{m \in \mathbb{N}^{*}}$ and $\left(P_{m}^{(m)}\right)_{m \in \mathbb{N}^{*}}$. Observe that $F P=D W A M_{0}$ and $L P=D W A M_{1}$.

- The minimum aggregator MIN and the maximum aggregator MAX are the aggregators $\left(M I N^{(m)}\right)_{m \in \mathbb{N}^{*}}$ and $\left(M A X^{(m)}\right)_{m \in \mathbb{N}^{*}}$.

- For any sequence $N=\left(N^{(m)}\right)_{m \in \mathbb{N}^{*}}$ of nonempty subsets $N^{(m)} \subseteq N_{m}$, the partial minimum aggregator $M I N_{N}$ and the partial maximum aggregator $M A X_{N}$, both associated to $N$, are the aggregators $\left(M I N_{N^{(m)}}^{(m)}\right)_{m \in \mathbb{N}^{*}}$ and $\left(M A X_{N^{(m)}}^{(m)}\right)_{m \in \mathbb{N}^{*}}$.

The next theorems describe the families of aggregators $M$ defined on $\bigcup_{m \geq 1} \mathbb{I}^{m}$ and fulfilling (In, SPL, A), (In, SPL, D), (In, SPL, SD) and (In, SPL, SB) respectively.

Theorem 5.1 The aggregator $M$ defined on $\bigcup_{m \geq 1} \mathbb{I}^{m}$ fulfils (In, SPL, A) if and only if

$$
M \in\{M I N, M A X, F P, L P\} .
$$

Proof. (Sufficiency). Trivial.

(Necessity). We construct the sequence $\left(M^{(m)}\right)_{m \in \mathbb{N}^{*}}$ by induction on $m$. The functions $M^{(2)}$ are given by Theorem 4.1. Thus assume that $M^{(k)}=\mathrm{MIN}^{(k)} \forall k \leq m$ for a fixed $m \geq 2$. By (A), we simply have

$$
M^{(m+1)}\left(x_{1}, \ldots, x_{m+1}\right)=M^{(2)}\left(M^{(m)}\left(x_{1}, \ldots, x_{m}\right), x_{m+1}\right)=\min _{i \in N_{m+1}} x_{i} .
$$

The other cases can be treated similarly.

Lemma 5.1 If the aggregator $M$ defined on $\bigcup_{m \geq 1} \mathbb{I}^{m}$ fulfils (In, SPL, D) then

$$
M^{(2)} \in\left\{W A M_{(1-\theta, \theta)}^{(2)}, O W A_{(1-\theta, \theta)}^{(2)} \mid \theta \in \mathbb{I}\right\} .
$$

Proof. Set $\theta=M^{(2)}(0,1)$ and $\bar{\theta}=M^{(2)}(1,0)$. By Proposition 4.1, we only have to prove that $\theta=\bar{\theta}$ or $\theta+\bar{\theta}=1$. Let us proceed in two steps:

(i) We have successively

$$
M^{(3)}(0,0,1) \stackrel{(D)}{=} M^{(3)}(0, \theta, \theta) \stackrel{(S P L)}{=} \theta M^{(3)}(0,1,1)
$$

and

$$
M^{(3)}(0,1,1) \stackrel{(D)}{=} M^{(3)}(\theta, \theta, 1) \stackrel{(S P L)}{=} \theta+(1-\theta) M^{(3)}(0,0,1) .
$$

It follows that

$$
M^{(3)}(0,0,1)=\frac{\theta^{2}}{\theta^{2}-\theta+1}
$$

and, similarly,

$$
M^{(3)}(1,0,0)=\frac{\bar{\theta}^{2}}{\bar{\theta}^{2}-\bar{\theta}+1} .
$$

(ii) We have, using (D) and (SPL),

$$
M^{(3)}(0,1,0)=\theta M^{(3)}(1,1,0)=\bar{\theta} M^{(3)}(0,1,1) .
$$

By $(i)$, the last equality becomes

$$
\theta\left(\bar{\theta}+(1-\bar{\theta}) \frac{\bar{\theta}^{2}}{\bar{\theta}^{2}-\bar{\theta}+1}\right)=\bar{\theta}\left(\theta+(1-\theta) \frac{\theta^{2}}{\theta^{2}-\theta+1}\right)
$$


or, after reduction, $\theta=0$ or $\bar{\theta}=0$ or $\theta=\bar{\theta}$ or $\theta+\bar{\theta}=1$. If $\theta=0$, i.e. $M^{(3)}(0,0,1)=0$, we have successively

$$
\bar{\theta} \stackrel{(S P L)}{=} M^{(3)}(\bar{\theta}, \bar{\theta}, 1) \stackrel{(D)}{=} M^{(3)}(1,0,1) \stackrel{(D)}{=} M^{(3)}(1,0,0)=\frac{\bar{\theta}^{2}}{\bar{\theta}^{2}-\bar{\theta}+1}
$$

and thus $\bar{\theta}=0$ or $\bar{\theta}=1$. We proceed similarly if $\bar{\theta}=0$.

Lemma 5.2 If the aggregator $M$ defined on $\bigcup_{m \geq 1} \mathbb{I}^{m}$ fulfils (D) and if $M^{(2)}$ fulfils (Sy) then $M$ fulfils (Sy).

Proof. Let us proceed by induction on $m \geq 2$. Assume that $M^{(m)}$ fulfils (Sy) for a fixed $m \geq 2$. By (D), we have

$$
\begin{aligned}
M^{(m+1)}\left(x_{1}, \ldots, x_{m+1}\right) & =M^{(m+1)}\left(x_{1}, m \cdot M^{(m)}\left(x_{2}, \ldots, x_{m+1}\right)\right) \\
& =M^{(m+1)}\left(m \cdot M^{(m)}\left(x_{1}, \ldots, x_{m}\right), x_{m+1}\right) .
\end{aligned}
$$

We can readily see that any permutation of indices of $x_{1}, \ldots, x_{m+1}$ keeps the left-hand side unchanged: indeed, this is true for all transpositions $T_{i j}(i, j=1, \ldots, m+1)$ including

$$
T_{1, m+1}=T_{1,2} T_{2, m+1} T_{1,2},
$$

where $T_{i j}$ denotes the transposition of $i$ and $j(i, j=1, \ldots, m+1)$.

Lemma 5.3 If the aggregator $M$ defined on $\bigcup_{m \geq 1} \mathbb{I}^{m}$ fulfils $\left(\right.$ Sy, I, D) then $M^{(2)}$ fulfils (B).

Proof. Let $\left(x_{1}, x_{2}, x_{3}, x_{4}\right) \in \mathbb{I}^{4}$. We have

$$
\begin{aligned}
& M^{(4)}\left(x_{1}, x_{2}, x_{3}, x_{4}\right) \stackrel{(D)}{=} \quad M^{(4)}\left[2 \cdot M^{(2)}\left(x_{1}, x_{2}\right), 2 \cdot M^{(2)}\left(x_{3}, x_{4}\right)\right] \\
& \stackrel{(\text { Sy })}{=} M^{(4)}\left[M^{(2)}\left(x_{1}, x_{2}\right), M^{(2)}\left(x_{3}, x_{4}\right), M^{(2)}\left(x_{1}, x_{2}\right), M^{(2)}\left(x_{3}, x_{4}\right)\right] \\
& \stackrel{(D)}{=} \quad M^{(4)}\left[4 \cdot M^{(2)}\left(M^{(2)}\left(x_{1}, x_{2}\right), M^{(2)}\left(x_{3}, x_{4}\right)\right)\right] \\
& \stackrel{(I)}{=} \quad M^{(2)}\left(M^{(2)}\left(x_{1}, x_{2}\right), M^{(2)}\left(x_{3}, x_{4}\right)\right)
\end{aligned}
$$

and the first expression is symmetric.

Theorem 5.2 (i) The aggregator $M$ defined on $\bigcup_{m \geq 1} \mathbb{I}^{m}$ fulfils (In, SPL, D) if and only if

$$
M \in\{M I N, M A X\} \cup\left\{D W A M_{\theta} \mid \theta \in \mathbb{I}\right\} .
$$

(ii) The aggregator $M$ defined on $\bigcup_{m \geq 1} \mathbb{I}^{m}$ fulfils (In, SPL, SD) if and only if

$$
M \in\{M I N, M A X, F P, L P, A M\} .
$$

Proof. (Sufficiency). We can easily check that all the aggregators mentioned in the statement fulfil the corresponding properties.

(Necessity). (i) Set $\theta=M^{(2)}(0,1)$ and $\bar{\theta}=M^{(2)}(1,0)$. By Lemma 5.1 , we have

$$
M^{(2)}=\operatorname{WAM}_{(1-\theta, \theta)}^{(2)} \text { or } \operatorname{OWA}_{(1-\theta, \theta)}^{(2)} .
$$


(i.1) Assume first that $M^{(2)}=\operatorname{WAM}_{(1-\theta, \theta)}^{(2)}$. Let us prove by induction on $m \geq 2$ that

$$
M^{(m)}\left(x_{1}, \ldots, x_{m}\right)=\frac{1}{\Delta_{m}} \sum_{i=1}^{m}(1-\theta)^{m-i} \theta^{i-1} x_{i} \forall\left(x_{1}, \ldots, x_{m}\right) \in \mathbb{I}^{m},
$$

where $\Delta_{m}=\sum_{i=1}^{m}(1-\theta)^{m-i} \theta^{i-1}$. The result holds true for $m=2$. Suppose it holds for a fixed $m \geq 2$ and show it still holds for $m+1$. Let $\left(x_{1}, \ldots, x_{m+1}\right) \in \mathbb{I}^{m+1}$ and set $x:=\left(x_{1}, \ldots, x_{m}\right)$. We then have

$$
\begin{aligned}
M^{(m+1)}\left(x_{1}, \ldots, x_{m+1}\right) & \stackrel{(D)}{=} \quad M^{(m+1)}\left(m \cdot M^{(m)}(x), x_{m+1}\right) \\
\stackrel{(S P L)}{=} & \begin{cases}\left(M^{(m)}(x)-x_{m+1}\right) \bar{\theta}_{m+1}^{(m+1)}+x_{m+1} & \text { if } x_{m+1} \leq M^{(m)}(x), \\
\left(x_{m+1}-M^{(m)}(x)\right) \theta_{m+1}^{(m+1)}+M^{(m)}(x) & \text { if } x_{m+1} \geq M^{(m)}(x)\end{cases}
\end{aligned}
$$

Let us show that $\bar{\theta}_{m+1}^{(m+1)}$ is uniquely determined. The same can be done for $\theta_{m+1}^{(m+1)}$. Using induction, we have

$$
\bar{\theta}_{m+1}^{(m+1)} \stackrel{(D)}{=} M^{(m+1)}\left(1, m \cdot \bar{\theta}_{m}^{(m)}\right) \stackrel{(i n d .)}{=} M^{(m+1)}\left(1, m \cdot\left(1-\frac{\theta^{m-1}}{\Delta_{m}}\right)\right)
$$

and

$$
\theta_{1}^{(m+1)} \stackrel{(D)}{=} M^{(m+1)}\left(m \cdot \theta_{1}^{(m)}, 0\right) \stackrel{(i n d .)}{=} M^{(m+1)}\left(m \cdot \frac{(1-\theta)^{m-1}}{\Delta_{m}}, 0\right) .
$$

Hence, using (SPL), we have the linear system

$$
\begin{aligned}
\bar{\theta}_{m+1}^{(m+1)} & =\frac{\theta^{m-1}}{\Delta_{m}} \theta_{1}^{(m+1)}+\left(1-\frac{\theta^{m-1}}{\Delta_{m}}\right) \\
\theta_{1}^{(m+1)} & =\frac{(1-\theta)^{m-1}}{\Delta_{m}} \bar{\theta}_{m+1}^{(m+1)}
\end{aligned}
$$

whose determinant

$$
\operatorname{det}\left(\begin{array}{cc}
1 & -\frac{\theta^{m-1}}{\Delta_{m}} \\
-\frac{(1-\theta)^{m-1}}{\Delta_{m}} & 1
\end{array}\right)=1-\frac{(1-\theta)^{m-1} \theta^{m-1}}{\Delta_{m}^{2}}
$$

is strictly positive. Indeed, we have $\Delta_{m} \geq(1-\theta)^{m-1}$ and $\Delta_{m} \geq \theta^{m-1}$, with at least one strict inequality. Consequently, $M^{(m+1)}\left(x_{1}, \ldots, x_{m+1}\right)$ is uniquely determined and $M$ must coincide with $\operatorname{DWAM}_{\theta}$.

$(i .2)$ Now assume that $M^{(2)}=\mathrm{OWA}_{(1-\theta, \theta)}^{(2)}$. Using Lemma 5.2, Lemma 5.3 and Corollary 4.2 successively, we have

$$
M^{(2)}=\mathrm{MIN}^{(2)} \text { or } \mathrm{MAX}^{(2)} \text { or } \mathrm{AM}^{(2)} .
$$

The function $\mathrm{AM}^{(2)}$ is a particular case of $(i .1)$ for which $\theta=1 / 2$. Let us show that if $M^{(2)}=\mathrm{MIN}^{(2)}$ then $M=\mathrm{MIN}$. The case $\mathrm{MAX}^{(2)}$ can be treated similarly. Let $m \geq 2$ and $\left(x_{1}, \ldots, x_{m}\right) \in \mathbb{I}^{m}$. Let us proceed in two steps:

(a) If $\min _{i \in N_{m}} x_{i}=0$ then, we have

$$
M^{(m)}\left(x_{1}, \ldots, x_{m}\right)=M^{(m)}\left(x_{1}, \ldots, 0, \ldots, x_{m}\right) \stackrel{(D)}{=} M^{(m)}(0, \ldots, 0) \stackrel{(I)}{=} 0 .
$$

(b) In the general case, we have

$$
M^{(m)}\left(x_{1}, \ldots, x_{m}\right) \stackrel{(S P L)}{=} M^{(m)}\left(x_{1}-x_{(1)}, \ldots, x_{m}-x_{(1)}\right)+x_{(1)} \stackrel{(a)}{=} x_{(1)}=\min _{i \in N_{m}} x_{i}
$$


since $\min _{i \in N_{m}}\left(x_{i}-x_{(1)}\right)=x_{(1)}-x_{(1)}=0$.

(ii) Suppose $M \notin\{$ MIN, MAX $\}$. Since (SD) trivially implies (D), by $(i)$, there exists $\theta \in \mathbb{I}$ such that

$$
M=\operatorname{DWAM}_{\theta}
$$

Since $M$ fulfils (SD), we must have

$$
M^{(3)}\left(M^{(2)}\left(x_{1}, x_{3}\right), x_{2}, M^{(2)}\left(x_{1}, x_{3}\right)\right)=M^{(3)}\left(x_{1}, x_{2}, x_{3}\right)
$$

for all $\left(x_{1}, x_{2}, x_{3}\right) \in \mathbb{I}^{3}$. In particular, for $\left(x_{1}, x_{2}, x_{3}\right)=e_{3}^{(3)}$, we have $M^{(3)}(\theta, 0, \theta)=M^{(3)}\left(e_{3}^{(3)}\right)$, that is, $(1-\theta)^{2} \theta+\theta^{3}=\theta^{2}$. Hence, we have $\theta \in\{0,1,1 / 2\}$ that allows to complete the proof.

Lemma 5.4 If the aggregator $M$ defined on $\bigcup_{m \geq 1} \mathbb{I}^{m}$ fulfils (In, SPL, SB) and if there exists $p \in \mathbb{N}^{*}, p \geq 2$ such that

$$
M^{(p)} \in\left\{W A M_{\omega^{(p)}}^{(p)} \mid \omega^{(p)} \in \mathbb{I}^{p}\right\} \backslash\left\{P_{i}^{(p)} \mid i \in N_{p}\right\}
$$

then

$$
M \in\left\{W A M_{\omega} \mid \omega=\left(\omega^{(m)} \in \mathbb{I}^{m}\right)_{m \in \mathbb{N}^{*}}\right\}
$$

Proof. There exists $\omega^{(p)} \in \mathbb{I}^{p}$ and $\left\{i_{1}, i_{2}\right\} \subseteq N_{p}$ with $i_{1} \neq i_{2}$, such that $M^{(p)}=\mathrm{WAM}_{\omega(p)}^{(p)}$ with $\omega_{i_{1}}, \omega_{i_{2}} \neq 0$.

Let $m \in \mathbb{N}^{*}, m \geq 2$, and let us show that $M^{(m)}$ is additive. Let $\left(u_{1}, \ldots, u_{m}\right),\left(v_{1}, \ldots, v_{m}\right) \in \mathbb{I}^{m}$ with $u_{i}+v_{i} \in \mathbb{I} \forall i \in N_{m}$. As in Lemma 4.1, we can assume $u_{i}, v_{i} \leq \inf \left\{\omega_{i_{1}}, \omega_{i_{2}}\right\} \in(0,1) \forall i \in N_{m}$. Consider the matrix $X$ of $p$ rows $r_{i}\left(i \in N_{p}\right)$ and $m$ columns $c_{j}\left(j \in N_{m}\right)$, where $r_{i}$ is defined as follows (for $i \in N_{p}$ ):

$$
r_{i}= \begin{cases}\frac{1}{\omega_{i_{1}}}\left(u_{1}, \ldots, u_{m}\right) & \text { if } i=i_{1}, \\ \frac{1}{\omega_{i_{2}}}\left(v_{1}, \ldots, v_{m}\right) & \text { if } i=i_{2}, \\ (m \cdot 0) & \text { if } i \in N_{p} \backslash\left\{i_{1}, i_{2}\right\} .\end{cases}
$$

Since $M$ fulfils (SPL, SB), we have

$$
M^{(m)}\left(u_{1}+v_{1}, \ldots, u_{m}+v_{m}\right)=M^{(m)}\left(u_{1}, \ldots, u_{m}\right)+M^{(m)}\left(v_{1}, \ldots, v_{m}\right) .
$$

We then can conclude as in the proof of Theorem 4.3.

Lemma 5.5 If the aggregator $M$ defined on $\bigcup_{m \geq 1} \mathbb{I}^{m}$ fulfils (In, SPL, SB) and if there exists $p \in \mathbb{N}^{*}, p \geq 2$ such that

$$
\begin{gathered}
M^{(p)} \in\left\{M I N_{N^{(p)}}^{(p)} \mid N^{(p)} \subseteq N_{p}\right\} \backslash\left\{P_{i}^{(p)} \mid i \in N_{p}\right\} \\
\left(\text { resp. } M^{(p)} \in\left\{M A X_{N^{(p)}}^{(p)} \mid N^{(p)} \subseteq N_{p}\right\} \backslash\left\{P_{i}^{(p)} \mid i \in N_{p}\right\}\right)
\end{gathered}
$$

then

$$
\begin{gathered}
M \in\left\{M I N_{N} \mid N=\left(N^{(m)} \subseteq N_{m}\right)_{m \in \mathbb{N}^{*}}\right\} \\
\left(\text { resp. } M \in\left\{M A X_{N} \mid N=\left(N^{(m)} \subseteq N_{m}\right)_{m \in \mathbb{N}^{*}}\right\}\right) .
\end{gathered}
$$

Proof. Assume that there exists $N^{(p)} \subseteq N_{p}$ and $\left\{i_{1}, i_{2}\right\} \subseteq N_{p}$ with $i_{1} \neq i_{2}$, such that $M^{(p)}=\operatorname{MIN}_{N^{(p)}}^{(p)}$. The case $\operatorname{MAX}_{N^{(p)}}^{(p)}$ can be treated similarly. 
Let $m \in \mathbb{N}^{*}, m \geq 2$, and $\left(u_{1}, \ldots, u_{m}\right),\left(v_{1}, \ldots, v_{m}\right) \in \mathbb{I}^{m}$. Next, consider the matrix $X$ of $p$ rows $r_{i}\left(i \in N_{p}\right)$ and $m$ columns $c_{j}\left(j \in N_{m}\right)$, where $r_{i}$ is defined as follows (for $\left.i \in N_{p}\right)$ :

$$
r_{i}= \begin{cases}\left(u_{1}, \ldots, u_{m}\right) & \text { if } i=i_{1} \\ \left(v_{1}, \ldots, v_{m}\right) & \text { if } i=i_{2}, \\ (m \cdot 1) & \text { if } i \in N_{p} \backslash\left\{i_{1}, i_{2}\right\}\end{cases}
$$

Since $M$ fulfils (SB), we have

$$
M^{(m)}\left(\min \left(u_{1}, v_{1}\right), \ldots, \min \left(u_{m}, v_{m}\right)\right)=\min \left(M^{(m)}\left(u_{1}, \ldots, u_{m}\right), M^{(m)}\left(v_{1}, \ldots, v_{m}\right)\right) .
$$

Using this argument, we can show that, if $\left(x_{1}, \ldots, x_{m}\right) \in \mathbb{I}^{m}$,

$$
M^{(m)}\left(x_{1}, \ldots, x_{m}\right)=\min _{i \in N_{m}} M^{(m)}\left(x_{i} e_{i}^{(m)}+\bar{e}_{i}^{(m)}\right) \stackrel{(S P L)}{=} \min _{i \in N_{m}}\left[\left(1-x_{i}\right) \bar{\theta}_{i}^{(m)}+x_{i}\right]
$$

Let us show that $\bar{\theta}_{i}^{(m)} \in\{0,1\} \forall i \in N_{m}$. Suppose it is not true. Since $M^{(m)}$ fulfils (In, SPL, B), by Lemma $4.1, M^{(m)}$ is additive and thus

$$
M^{(m)} \in\left\{\mathrm{WAM}_{\omega(m)}^{(m)} \mid \omega^{(m)} \in \mathbb{I}^{m}\right\} \backslash\left\{\mathrm{P}_{i}^{(m)} \mid i \in N_{m}\right\}
$$

which is impossible by Lemma 5.4. Indeed, we have

$$
\left\{\mathrm{WAM}_{\omega^{(p)}}^{(p)} \mid \omega^{(p)} \in \mathbb{I}^{p}\right\} \cap\left\{\operatorname{MIN}_{N^{(p)}}^{(p)} \mid N^{(p)} \subseteq N_{p}\right\}=\left\{\mathrm{P}_{i}^{(p)} \mid i \in N_{p}\right\}
$$

since if $M^{(p)}$ belongs to the left-hand set then we necessarily have $\omega_{i}^{(p)}=\theta_{i}^{(p)} \in\{0,1\}$.

Finally, we have $M^{(m)}=\operatorname{MIN}_{N^{(m)}}^{(m)}$ with $N^{(m)}=\left\{i \in N_{m} \mid \bar{\theta}_{i}^{(m)}=0\right\}$.

Theorem 5.3 The aggregator $M$ defined on $\bigcup_{m \geq 1} \mathbb{I}^{m}$ fulfils (In, SPL, SB) if and only if

$$
M \in\left\{\operatorname{MIN}_{N}, \operatorname{MAX}_{N} \mid N=\left(N^{(m)} \subseteq N_{m}\right)_{m \in \mathbb{N}^{*}}\right\} \cup\left\{W A M_{\omega} \mid \omega=\left(\omega^{(m)} \in \mathbb{I}^{m}\right)_{m \in \mathbb{N}^{*}}\right\} .
$$

Proof. (Sufficiency). We can easily check that all the aggregators mentioned in the statement fulfil (In, SPL, SB).

(Necessity). If, for all $m \in \mathbb{N}^{*}, M^{(m)} \in\left\{\mathrm{P}_{i}^{(m)} \mid i \in N_{m}\right\}$ then we can conclude immediately. Otherwise, there exists $p \in \mathbb{N}^{*}, p \geq 2$ such that $M^{(p)} \notin\left\{\mathrm{P}_{i}^{(p)} \mid i \in N_{p}\right\}$. Since $M^{(p)}$ fulfils (In, SPL, B) then, by Theorem $4.3, M^{(p)}$ fulfils the assumptions of Lemma 5.4 or 5.5 , and we can conclude again.

To summarize this section, we present a table containing the above characterizations (Table 3). The table also contains some corollaries which can be checked easily. In particular, we obtain a complete characterization of the weighted arithmetic mean aggregators. Moreover, AM could be isolated using a property of strict increasing monotonicity.

\section{Some related aggregation functions}

From the stability properties introduced in this paper, we can derive a variety of stability properties by using automorphisms.

Definition 6.1 A continuous, strictly increasing function $\varphi:$ II $\rightarrow$ II with boundary conditions $\varphi(0)=0, \varphi(1)=1$ is called an automorphism of the interval $\mathbb{I}$. 


\begin{tabular}{|c|c|c|}
\hline In, SPL, A & Sy, In, SPL, A & In, SSI, SSN, A \\
\hline MIN, MAX, FP, LP. & MIN, MAX. & FP, LP. \\
\hline In, SPL, D & Sy, In, SPL, D & In, SSI, SSN, D \\
\hline MIN, MAX, $\left\{\right.$ DWAM $\left._{\theta} \mid \theta \in \mathbb{I}\right\}$. & MIN, MAX, AM. & $\left\{\operatorname{DWAM}_{\theta} \mid \theta \in \mathbb{I}\right\}$ \\
\hline In, SPL, SD & Sy, In, SPL, SD & In, SSI, SSN, SD \\
\hline MIN, MAX, FP, LP, AM. & MIN, MAX, AM. & FP, LP, AM. \\
\hline In, SPL, SB & Sy, In, SPL, SB & In, SSI, SSN, SB \\
\hline $\begin{array}{l}\left\{\operatorname{MIN}_{N}, \operatorname{MAX}_{N} \mid N=\left(N^{(m)} \subseteq N_{m}\right)_{m \in \mathbb{N}^{*}}\right\} \\
\left\{\operatorname{WAM}_{\omega} \mid \omega=\left(\omega^{(m)} \in \mathbb{I}^{m}\right)_{m \in \mathbb{N}^{*}}\right\}\end{array}$ & MIN, MAX, AM. & $\left\{\mathrm{WAM}_{\omega} \mid \omega=\left(\omega^{(m)} \in \mathbb{I}^{m}\right)_{m \in \mathbb{N}^{*}}\right\}$ \\
\hline
\end{tabular}

Table 3: Results from Section 5

The next two definitions introduce some new stability properties with the help of automorphisms.

Definition 6.2 Given an automorphism $\varphi$ of $\mathbb{I}$, the aggregation function $M^{(m)}$ defined on $\mathbb{I}^{m}$ is said to be stable for the admissible $\varphi$-similarities $\left(\mathrm{SSI}_{\varphi}\right)$ (resp. stable for the admissible $\varphi$ translations $\left(\operatorname{STR}_{\varphi}\right)$, stable for the admissible positive $\varphi$-linear transformations $\left(\mathrm{SPL}_{\varphi}\right)$, stable for the $\varphi$-negation $\left(\operatorname{SSN}_{\varphi}\right)$ ) if the aggregation function $F^{(m)}$ defined on $\mathbb{I}^{m}$ by

$$
F^{(m)}\left(x_{1}, \ldots, x_{m}\right)=\varphi M^{(m)}\left(\varphi^{-1} x_{1}, \ldots, \varphi^{-1} x_{m}\right) \forall\left(x_{1}, \ldots, x_{m}\right) \in \mathbb{I}^{m}
$$

fulfils (SSI) (resp. (STR), (SPL), (SSN)).

Definition 6.3 Given an automorphism $\varphi$ of $\mathbb{I}$, the aggregator $M$ defined on $\bigcup_{m \geq 1} \mathbb{I}^{m}$ fulfils $\left(S S I_{\varphi}\right)$ (resp. $\left.\left(S T R_{\varphi}\right),\left(S P L_{\varphi}\right),\left(S S N_{\varphi}\right)\right)$ if, for all $m \in \mathbb{N}^{*}$, the aggregation function $M^{(m)}$ fulfils $\left(S S I_{\varphi}\right)\left(\operatorname{resp} .\left(S T R_{\varphi}\right),\left(S P L_{\varphi}\right),\left(S S N_{\varphi}\right)\right)$.

Given an automorphism $\varphi$ of II and an aggregation function $M^{(m)}$ defined on $\mathbb{I}^{m}$, we let $M_{\varphi}^{(m)}$ denote an aggregation function defined on $\mathbb{I}^{m}$ by

$$
M_{\varphi}^{(m)}\left(x_{1}, \ldots, x_{m}\right)=\varphi^{-1} M^{(m)}\left(\varphi x_{1}, \ldots, \varphi x_{m}\right) \forall\left(x_{1}, \ldots, x_{m}\right) \in \mathbb{I}^{m} .
$$

Likewise, given an automorphism $\varphi$ of $\mathbb{I}$ and an aggregator $M$ defined on $\bigcup_{m>1} \mathbb{I}^{m}$, we let $M_{\varphi}$ denote the aggregator defined on $\bigcup_{m \geq 1} \mathbb{I}^{m}$ and whose $m$ th aggregation function $\left(m \in \mathbb{N}^{*}\right)$ is $M_{\varphi}^{(m)}$. We call $M_{\varphi}^{(m)}$ (resp. $M_{\varphi}$ ) the $\varphi$-transform of $M^{(m)}$ (resp. $M$ ). We then have the following result which can be easily checked.

Proposition 6.1 Let $\varphi$ be an automorphism of II and let $P \in\{S S I, S T R, S P L, S S N\}, Q \in\{S y$, In, $A, A D, B\}, R \in\{S y$, In, $A, D, S D, S B\}$. Then

1. Let $m \in \mathbb{N}^{*}$. The aggregation function $M^{(m)}$ defined on $\mathbb{I}^{m}$ fulfils $(P)$ (resp. $(Q)$ ) if and only if the aggregation function $M_{\varphi}^{(m)}$ defined on $\mathbb{I}^{m}$ fulfils $\left(P_{\varphi}\right)$ (resp. (Q)).

2. The aggregator $M$ defined on $\bigcup_{m \geq 1} \mathbb{I}^{m}$ fulfils $(P)$ (resp. $\left.(R)\right)$ if and only if the aggregator $M_{\varphi}$ defined on $\bigcup_{m \geq 1} \mathbb{I}^{m}$ fulfils $\left(P_{\varphi}\right)$ (resp. $(R)$ ).

The previous proposition allows to generate a lot of characterizations from those we obtained. For instance, if $\varphi(x)=x^{2}$ on II, we have the following result. 
Corollary 6.1 Let $m \in \mathbb{N}^{*}, m \geq 2$. The aggregation function $M^{(m)}$ defined on $\mathbb{I}^{m}$ fulfils (Sy, In, $\left.S P L_{\varphi}, B\right)$ with $\varphi(x)=x^{2}$ if and only if

$$
M^{(m)} \in\left\{M I N^{(m)}, M A X^{(m)}, A M_{\varphi}^{(m)}\right\}
$$

where

$$
A M_{\varphi}^{(m)}\left(x_{1}, \ldots, x_{m}\right)=\sqrt{\frac{1}{m} \sum_{i=1}^{m} x_{i}^{2}} \forall\left(x_{1}, \ldots, x_{m}\right) \in \mathbb{I}^{m}
$$

\section{$7 \quad$ Aggregation functions defined on real intervals containing $\mathbb{I}^{m}$}

Let $\Omega$ be any interval such that $\mathbb{I} \subseteq \Omega \subseteq \mathbb{R}$. Obviously, all the definitions and properties introduced earlier can be defined on $\Omega$ rather than $\mathbb{I}$. In this section, we show that all the results obtained so far can be adapted to aggregation functions defined on $\Omega^{m}$ and aggregators defined on $\bigcup_{m \geq 1} \Omega^{m}$.

The next two results establish a link between some aggregation functions defined on $\Omega^{m}$ and their restrictions to $\mathbb{I}^{m}$.

Proposition 7.1 Let $P \in\{S y, I n, C o m p, I, S S I, S T R, S P L, S S N\}, Q \in\{A, A D, B\}, R \in\{A, D$, $S D, S B\}$. Then

1. Let $m \in \mathbb{N}^{*}$. If the aggregation function $F^{(m)}$ defined on $\Omega^{m}$ fulfils $(P)($ resp. (Comp, Q)) then its restriction $M^{(m)}$ to $\mathbb{I}^{m}$ fulfils $(P)$ (resp. (Comp, Q)).

2. If the aggregator $F$ defined on $\bigcup_{m \geq 1} \Omega^{m}$ fulfils $(P)$ (resp. (Comp, R)) then its restriction $M$ to $\bigcup_{m \geq 1} \mathbb{I}^{m}$ fulfils $(P)$ (resp. (Comp, R)).

Proof. Easy.

Proposition 7.2 Any aggregation function $F^{(m)}\left(m \in \mathbb{N}^{*}\right)$ defined on $\Omega^{m}$ and fulfilling (SPL) is completely defined by its restriction to $\mathbb{I}^{m}$. The same holds true for any aggregator $F$.

Proof. Let $M^{(m)}$ denote the restriction to $\mathbb{I}^{m}$ of $F^{(m)}$. By (SPL) we have, for all $\left(x_{1}, \ldots, x_{m}\right) \in$ $\Omega^{m}$,

$$
F^{(m)}\left(x_{1}, \ldots, x_{m}\right)=\left\{\begin{array}{l}
x \text { if }\left(x_{1}, \ldots, x_{m}\right)=(x, \ldots, x) \\
\left(x_{(m)}-x_{(1)}\right) M^{(m)}\left[\frac{x_{1}-x_{(1)}}{x_{(m)}-x_{(1)}}, \ldots, \frac{x_{m}-x_{(1)}}{x_{(m)}-x_{(1)}}\right]+x_{(1)} \text { otherwise }
\end{array}\right.
$$

We then can conclude.

Propositions 7.1 and 7.2 allow to obtain characterizations for aggregation functions defined on $\Omega^{m}$ or aggregators defined on $\bigcup_{m \geq 1} \Omega^{m}$. For instance, we have the following:

Corollary 7.1 Let $m \in \mathbb{N}^{*}, m \geq 2$. The aggregation function $F^{(m)}$ defined on $\mathbb{R}^{m}$ fulfils (Sy, In, $S P L, B)$ if and only if

$$
F^{(m)} \in\left\{M I N^{(m)}, M A X^{(m)}, A M^{(m)}\right\}
$$

\section{Conclusion}

We have characterized some aggregation functions and some aggregators which can be useful in multicriteria decision making procedures. The results contribute to the theory of MCDM and can help the decision maker in choosing a particular family of functions on the basis of some properties expected in advance from an aggregation function.

\section{Acknowledgements}

The authors are indebted to Yves Crama and Marc Roubens for useful discussions. 


\section{References}

[1] J. Aczél (1948), On mean values, Bulletin of the American Math. Society, 54: 392-400.

[2] J. Aczél (1966), Lectures on Functional Equations and Applications, (Academic Press, NewYork).

[3] J. Aczél (1984), On weighted synthesis of judgements, Aequationes Mathematicae 27: 288307.

[4] J. Aczél and C. Alsina (1987), Synthesizing judgments: a functional equations approach, Math. Modelling, 9: 311-320.

[5] J. Aczél and F.S. Roberts (1989), On the possible merging functions, Math. Social Sciences, 17: 205-243.

[6] J. Aczél, F.S. Roberts and Z. Rosenbaum (1986), On scientific laws without dimensional constants, Journal of Math. Analysis and Appl., 119: 389-416.

[7] J. Fodor, J.-L. Marichal, On nonstrict means, Aequationes Mathematicae, in press.

[8] J. Fodor, J.-L. Marichal and M. Roubens (1994), Characterization of some aggregation functions arising from MCDM problems, Proceedings of IPMU'94, Paris: 1026-1031.

[9] J. Fodor and M. Roubens (1994), Fuzzy Preference Modelling and Multicriteria Decision Support, Kluwer, Dordrecht.

[10] A.N. Kolmogoroff (1930), Sur la notion de la moyenne, Accad. Naz. Lincei Mem. Cl. Sci. Fis. Mat. Natur. Sez.,12: 388-391.

[11] C.H. Ling (1965), Representation of associative functions, Publ. Math. Debrecen, 12: 189212.

[12] J.-L. Marichal and P. Mathonet, A characterization of the ordered weighted averaging functions based on the ordered bisymmetry property, IEEE Trans. Fuzzy Syst., submitted.

[13] J.-L. Marichal and M. Roubens (1993), Characterization of some stable aggregation functions, Proc. Intern. Conf. on Industrial Engineering and Production Management, Mons, Belgique: 187-196.

[14] M. Nagumo (1930), Über eine Klasse der Mittelwerte, Japanese Journal of Mathematics, 6: 71-79.

[15] F.S. Roberts (1979), Measurement Theory with Applications to Decision-making, Utility and the Social Sciences, Addison-Wesley Pub., Reading.

[16] W. Silvert (1979), Symmetric summation: A class of operations on fuzzy sets, IEEE Trans. Systems, Man Cybernet., 9: 657-659. 